\title{
Inversion analysis and safety evaluation of deformation of cutoff wall in Daning Reservoir
}

\author{
YUAN Hong-hu' ${ }^{1}$, Liao $\mathrm{Kun}^{2}$, Wang $\mathrm{Yu}^{2 *}$ \\ ${ }^{1}$ Beijing Institute of water, Beijing, 100048, China \\ 2. School of Mechanical Engineering, Xihua University
}

\begin{abstract}
There is a large amount of backfilled earth on both sides of the wall and the top of the lower wall of the Daning Reservoir's cutoff wall, which is greatly disturbed by the project, and the safety of the cutoff wall needs to be evaluated. Based on the deformation law and stress condition of rigid cutoff wall during construction, according to the connection characteristics of rigid cutoff wall and plastic cutoff wall structure, the stress analysis of rigid cutoff wall is carried out by using field measurement data, so as to deduce the deformation law of plastic cutoff wall underneath, and evaluate the safety of plastic cutoff wall. The results show that the plastic cutoff wall is mainly affected by compressive stress, and the maximum compressive stress is far less than the allowable compressive stress, which indicates that the plastic cutoff wall is safe and stable during the whole construction period, although it is affected by construction and rigid cutoff wall.
\end{abstract}

\section{Introduction}

The cutoff wall of Beijing Daning Reservoir is composed of plastic concrete wall ( lower wall ) buried underground and newly built reinforced concrete wall ( upper wall ). The backfill construction on both sides of the newly built upper wall not only has a mechanical effect on the upper wall, but also has influence on the stability and permeability of the lower wall.

Wen Lifeng et $\mathrm{al}^{[1]}$ studied the mechanical properties of concrete cutoff wall of dam foundation from the perspective of statistical analysis; Wu Mengxi, et a ${ }^{[2]}$ carried out dynamic coupling simulation analysis of seepage and stress deformation on upstream cofferdam of Lawa hydropower station; Zhang Biliang et $\mathrm{al}^{[3]}$ put forward the construction method of basalt fiber reinforced TRD cutoff wall by using TRD construction method of cement soil mixing method to break through the system; Wang Yuanming, et a ${ }^{[4]}$ proposed a detection method based on elastic wave image method for the integrity detection of cutoff wall; Xu Han, et al ${ }^{[5]}$ studied the influence of rigid-flexible normal contact on the stress of concrete cutoff wall; Liang Yan et $\mathrm{al}^{[6]}$ conducted numerical simulation analysis on the cutoff wall of dam foundation of Longkaikou Hydropower Station; Liu Haojie, et $\mathrm{al}^{[7]}$ took a reservoir as an example, and carried out seepage control and three-dimensional numerical analysis of deep overburden earth-rock dam; Chen Xiaolian et $\mathrm{al}^{[8]}$ based on the foundation pit pumping test of a hydropower station on the Dadu River in Sichuan Province; and judged the anti-seepage effect of the cutoff wall by calculating the parameters; Liu Guangming, et a ${ }^{[9]}$ based on the existing test and measured parameters, the three-dimensional numerical simulation of the construction effect of backfill soil on both sides of the cutoff wall of Daning Reservoir is carried out; Gao Jianglin et $\mathrm{al}^{[10]}$ used ABAQUS finite element software to establish the numerical model of seepage and stress coupling; Huang Chenjie, et $\mathrm{al}^{[11]}$ used the finite difference software FLAC3D to simulate the influence of the penetration depth of the suspended cutoff wall in the double-layer embankment foundation on the seepage deformation; Liu Guangming et $\mathrm{al}^{[12]}$ carried out real-time dynamic automatic continuous monitoring of various stress effects in the construction process of filling impervious wall.

\section{Project Overview}

Due to the reconstruction project of Jingshi Expressway (Fifth Ring Road Zhaoxindian Interchange), the Dujiakan toll station was expanded to the Daning Reservoir area. The expansion scope includes the pile number of the impervious wall that has been built in the reservoir XD0+697.2 XD0+835.9, length $138.7 \mathrm{~m}$. The intersection of road and cutoff wall affects the position as shown in Figure. $\left[^{[13]}\right.$. 


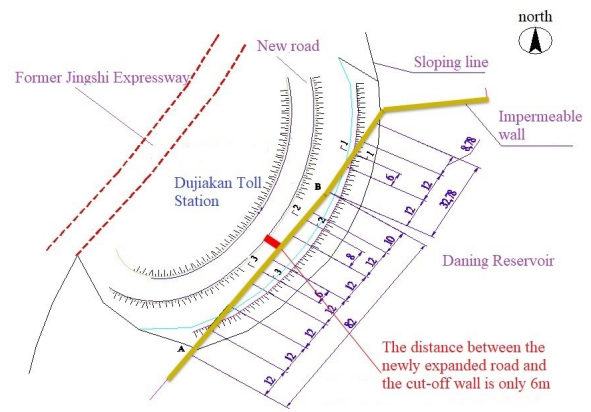

Fig. 1 plan of Beijing Shijiazhuang Expressway and reservoir cutoff wall

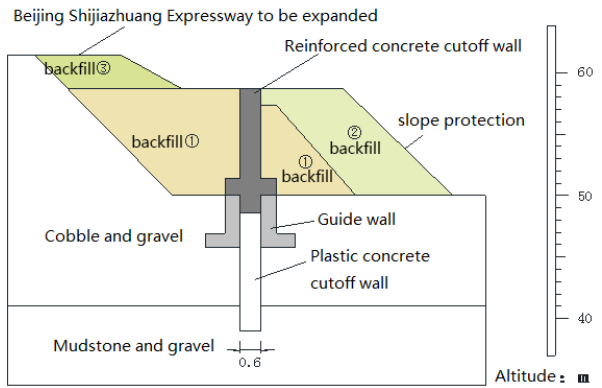

Fig. 2 structure of cutoff wall and sequence of backfill
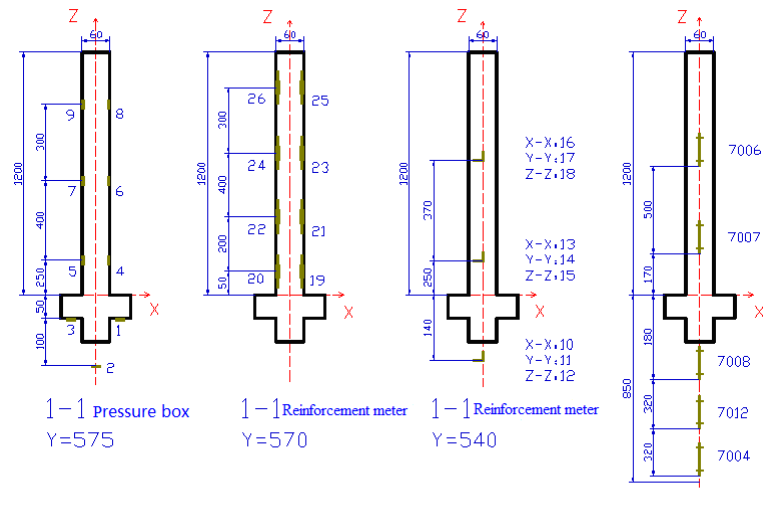

Fig. 3 Schematic diagram of installation position of instruments in the second monitoring plane

After the filling near the wall is completed; the reservoir area slightly far away from the wall shall be filled. The backfill sequence number represents the sequence of backfill, as shown in Figure 2.

The installation position of the second monitoring section test instrument is shown in Figure 3.

\section{Analysis of deformation of plastic seepage wall}

The stress and deformation of the plastic cut-off wall during construction are mainly caused by the upper steel cut-off wall and filling construction. The deformation of the plastic cut-off wall should be mainly lateral deformation, and its simplified analysis mechanical model is shown in Figure 4.

According to the stress condition of the plastic cutoff wall shown in Fig. 4, the deflection differential equation of the plastic cutoff wall can be written as

$$
E I_{y} \frac{d^{4} x(z, t)}{d z^{4}}+k_{x} x(z, t)=0
$$

The above formula can be simplified as

$$
\frac{d^{4} x(z, t)}{d z^{4}}+\lambda^{4} x(z, t)=0
$$

among $\lambda=\sqrt[4]{k_{x} / E I_{y}}, \quad E I_{y}$ Is the bending stiffness of the plastic cutoff wall, where Y-axis is the neutral axis.

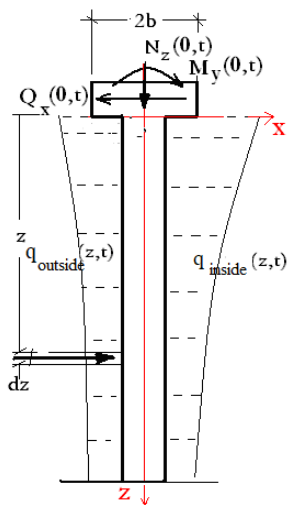

Fig. 4 mechanical model for deformation analysis of plastic cutoff wall

Due to the particularity of the project and the construction process, the inclinometer is only set in XD0 +712 (the second) plastic cutoff wall to monitor the deformation of the plastic cutoff wall.

$\mathrm{XD} 0+712$ (the second one) is shown in Fig. 3, and the layout of the three monitoring points is basically similar.

The location of inclinometer points of plastic cutoff wall is shown in Fig.3, and the construction disturbance monitoring curve and least square fitting curve are shown in Fig.5, the time $t$ is calculated from March 25, 2011 as the first day.

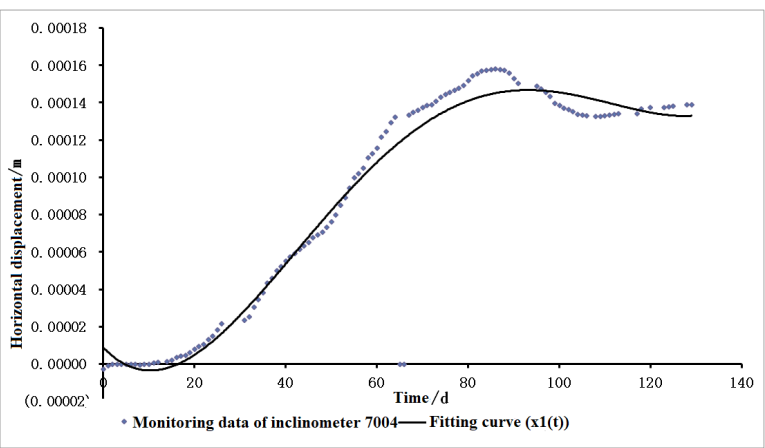

(a) 7004 monitoring point 


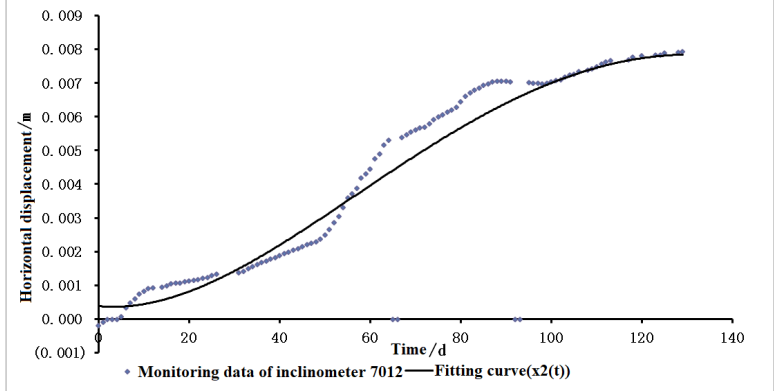

(b) 7012 monitoring point

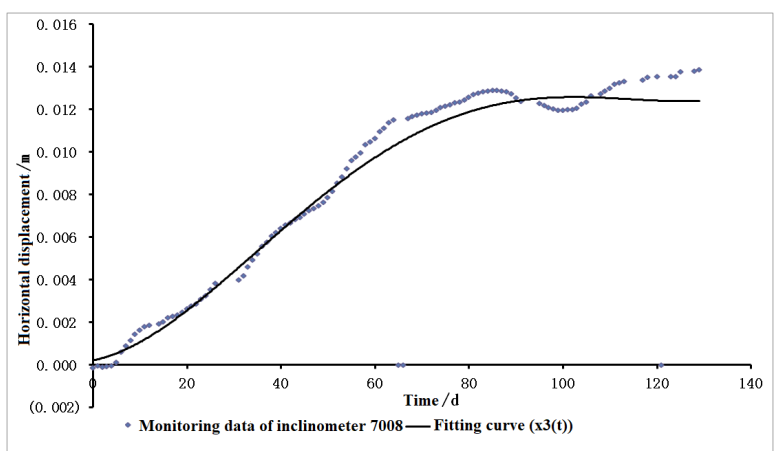

(c) 7008 monitoring point

Figure 5 inclinometer monitoring data and fitting curve

The corresponding least square polynomial monitoring fitting curve equation is

$x_{1}(t)=5 \times 10^{-12} t^{4}-2 \times 10^{-9} t^{3}+2 \times 10^{-7} t^{2}-3 \times 10^{-6} t+10^{-5}$

$x_{2}(t)=3 \times 10^{-11} t^{4}-2 \times 10^{-8} t^{3}+2 \times 10^{-6} t^{2}-2 \times 10^{-5} t+0.0004$

$x_{3}(t)=2 \times 10^{-10} t^{4}-6 \times 10^{-8} t^{3}+5 \times 10^{-6} t^{2}+3 \times 10^{-5} t+0.0002(3)$

As shown in Figure 3, the coordinates of the three monitoring points of the plastic cutoff wall are $z_{1}=1.8, z_{2}=5, z_{3}=8.2$ 。

The estimation expression of horizontal displacement monitoring result $x(z, t)$ can be obtained as follows

$$
\begin{aligned}
x(z, t)= & \left(5.4658-4.0179 z+0.708 z^{2}\right) \times 10^{-11} t^{4} \\
& +\left(-1.5430+1.6339 z-1.0742 z^{2}\right) \times 10^{-9} t^{3} \\
& +\left(-2.8516+1.6788 z+0.5860 z^{2}\right) \times 10^{-7} t^{2} \\
& +\left(3.6006-2.7536 z+0.32715 z^{2}\right) \times 10^{-5} t \\
& +\left(-4.6865+3.1793 z-0.2881 z^{2}\right) \times 10^{-4}
\end{aligned}
$$

Calculate the monitoring values of the corresponding three monitoring points at different times, replace it with the following, and solve the transcendental equation

$$
x(z, t)=\frac{e^{-\lambda z}}{2 \lambda^{3} E I_{y}}\left[\lambda M_{y}(0, t)(\cos \lambda z+\sin \lambda z)-Q_{x}(0, t) \cos \lambda z\right]
$$

The deformation series values of three monitoring points at different times are obtained. After combining with the above formula, the least square method is used to get the $x(z, t)$-estimation expression, and then the iterative method is used to get the value that makes the interpolation square of the two minimum by MATLAB. From this we can get the conclusion $\lambda=1.239$.
From the formula $\lambda=\sqrt[4]{k_{x} / E I_{y}}$, and pay attention to the elastic modulus of the plastic cutoff wall $E=2000 \mathrm{MPa}$, The section moment of inertia is $I_{y}=0.018 \mathrm{~m}^{4}$, and the horizontal resistance coefficient of the plastic cutoff wall is obtained from this inversion as

$$
k_{x}=84.8375 \mathrm{MPa} / \mathrm{m}
$$

\section{Stability evaluation of plastic cutoff wall}

The corresponding stress at each dangerous point is shown in table 1 .

It can be seen from the results in table 3.6 that the maximum equivalent stress at the dangerous point is only $0.7237 \mathrm{mpa}$; and the allowable stress $2 \mathrm{MPa}$ of cutoff wall; and all points of the plastic seepage wall are under pressure during the construction period. It is seen that the plastic seepage wall is safe and will not be damaged due to insufficient strength.

Table.1 stress of each dangerous point on dangerous section during construction of plastic cutoff wall / MPa

\begin{tabular}{ccccccc}
\hline section & point & $\sigma_{M z}$ & $\sigma_{N z}$ & $\sigma_{z}$ & $\tau_{x z}$ & $\sigma_{r q}$ \\
& & & & & & \\
\hline \multirow{2}{*}{1} & 1 & 0.326 & -0.398 & -0.071 & 0 & 0.071 \\
& 2 & 0 & -0.398 & -0.398 & -0.099 & 0.433 \\
& 3 & -0.326 & -0.398 & -0.724 & 0 & 0.724 \\
& 1 & 0 & -0.581 & -0.581 & 0 & 0.581 \\
\hline
\end{tabular}

\section{Conclusion}

The research work analyzes the deformation law of plastic cutoff wall under the influence of construction and rigid cutoff wall. It shows that although the plastic cutoff wall is affected by the construction and rigid cutoff wall; it is safe and stable during the whole construction period, which provides a theoretical basis for the construction safety of the construction personnel.

\section{References :}

1. Wen Lifeng, Li Yanlong, Chai Junrui. Statistical analysis of mechanical properties of concrete cutoff wall of dam foundation[J]. Journal of Hydraulic Engineering, 2021, 52(02): 241-254.

2. Wu Mengxi, Song Shixiong, Wu Wenhong. Dynamic coupling simulation analysis of seepage and stress deformation of upstream cofferdam of Lawa Hydropower Station[J]. Chinese Journal of Geotechnical Engineering, 2021, 43(04): 613-623.

3. Zhang Biliang, Zhang Long, He Peng, Wu Mimi, Wang Shengnian, Zhang Peng. The anti-hydraulic splitting performance of TRD cutoff wall reinforced with basalt fiber[J]. South-to-North Water Transfer 
and Water Conservancy Science and Technology (Chinese and English), 2021, 19 (02 ): 400-408.

4. Wang Yuanming, Yao Hailin, Che Ailan, Li Heng, Luo Xingwen, Lu Zheng. Rapid detection method and numerical simulation of the integrity of the impervious wall of a dyke[J]. Geotechnical Foundation, 2019, 33(03): 370-374.

5. Xu Han, Pan Jiajun, Jiang Jiju, Tan Fan, Yang Xinguang. Research on the influence of rigid and flexible normal contact on the stress of concrete cutoff walls[J]. Chinese Journal of Geotechnical Engineering, 2019, 41(S1): 121-124 .

6. Liang Yan, Mao Ruimin, Wang Yi, Luo Xiaoyong. Numerical simulation of anti-seepage wall of dam foundation of Longkaikou Hydropower Station[J]. Journal of Water Resources and Hydropower Engineering, 2018(03): 57-64.

7. Liu Haojie, Ren Jie, Yang Jie, Cheng Lin, Zhang Xiaofei. Seepage control and three-dimensional numerical analysis of earth-rock dams with deep overburden[J]. Journal of Water Resources and Water Engineering, 2018, 29(02): 219-224+ 228.

8. Chen Xiaolian, Article, Hu Jinshan, Min Yongzhang, Liang Xing, Sun Ronglin, Li Runchao. Application of Analytical and Numerical Methods in the Evaluation of the Effect of Impervious Walls in Hydropower Stations[J]. Earth Science, 2016, 41(04): 701- 710.

9. Liu Guangming, Yuan Honghu, Huang Weihong, Liu Miao, Wang Zhiyin. Numerical simulation of Daning Reservoir cutoff wall affected by side backfill construction[J]. Rock and Soil Mechanics, 2013, 34(06): 1786-1790+1798 .

10. Gao Jianglin, Chen Yunxiang. Numerical simulation of the interaction between the cutoff wall and the dam based on the coupling of seepage and stress[J]. Journal of Water Resources and Hydropower Engineering, 2013(02): 58-63.

11. Huang Chenjie, Wang Baotian. Numerical simulation of anti-seepage effect of suspended cutoff wall[J]. Hydropower Energy Science, 2013, 31(05): 123-125+95.

12. [Liu Guangming, Zhang Ruman, Liu Miao, Wang Zhiyin, Yuan Honghu. On-site monitoring of the impact of backfill construction on the cutoff wall of Daning Reservoir[J]. Water Resources and Hydropower Technology, 2013, 44(06): 68-72+76.

13. Beijing Institute of Water Conservancy Planning and Design. On-site monitoring and analysis of the impact of construction on the west embankment of Beijing Daning Reservoir [R]. Beijing: Beijing Institute of Water Conservancy Planning and Design, 2012. 